\title{
A Reappraisal of Sex-Specific Cancer Survival Trends Among Adolescents and Young Adults in the United States
}

\author{
Lihua Liu, Diana J. Moke, Kai-Ya Tsai, Amie Hwang, David R. Freyer, Ann S. Hamilton, \\ Juanjuan Zhang, Myles Cockburn, Dennis Deapen
}

See the Notes section for the full list of authors' affiliations.

Correspondence to: Lihua Liu, PhD, Los Angeles Cancer Surveillance Program, Department of Preventive Medicine, Keck School of Medicine, University of Southern California, 2001 N. Soto St, SSB-305E, Los Angeles, CA 90032 (e-mail: lihualiu@usc.edu).

\begin{abstract}
Background: Cancer survival among adolescents and young adults (AYAs) was previously reported as showing little or no improvement compared to younger or older counterparts. The role of the HIV/AIDS epidemic in the AYA survival deficit has not been evaluated.

Methods: Using cancer registry data from the Surveillance, Epidemiology, and End Results program (SEER 9), we examined sex-specific 5-year relative survival trends for children (0-14 years old), AYAs (15-39years old), and older adults (40 years and older) diagnosed with cancer during 1973-2009 and followed through the end of 2014. The analysis was conducted with and without Kaposi sarcoma (KS) and lymphomas, and by two time periods: 1973-1977 (before the human immunodeficiency virus/acquired immunodeficiency syndrome [HIV/AIDS] epidemic) and 2005-2009 (after the HIV/AIDS epidemic waned). Results: A total of 3209721 invasive cancer cases were included in the study (27646 children, 213930 AYAs, and 2968145 older adults; 24803 children, 178741 AYAs, and 2844062 older adults when KS and lymphoma cases were excluded). We found that 5-year relative survival for AYAs exceeded that of children and older adults before the onset of the HIV/AIDS epidemic (eg, during 1973-1979, 0.58-0.67 among male AYAs as compared with 0.47-0.61 for male children and 0.36-0.42 for male older adults; among female AYAs, the numbers were 0.73-0.77 as compared with 0.51-0.65 for female children and 0.520.55 for female older adults); substantially declined during 1983-1997 when HIV/AIDS lacked effective treatment among male AYAs; and returned to be higher than most age groups by the late 1990s after HIV/AIDS was controlled. Nonetheless, comparison of survival improvement between 1973-1977 and 2005-2009 demonstrated less progress in AYAs than other age groups, which was due to AYAs' better baseline survival and larger survival gains among children and older adults in recent years. Conclusions: Apart from the temporary impact of HIV/AIDS, survival among AYA cancer patients has shown sustained improvement and superiority relative to other age groups. However, these encouraging findings do not negate the distinctive challenges in cancer diagnosis, treatment, and survivorship faced by AYAs.
\end{abstract}

Cancer is the most common cause of death due to disease among adolescents and young adults (AYAs), defined by the US National Cancer Institute as 15-39years old (1). Each year about 70000 cancer cases are diagnosed among AYAs in the United States, approximately six times the incidence of cancer among children under age 15 (2). Furthermore, during a life stage characterized by dramatic physical and psychosocial development, AYA cancer patients experience a multitude of distinctive challenges, including differing cancer and host biology, delayed diagnosis, limited access to optimal treatment and ageappropriate services, excess treatment-related toxicity, and low levels of participation in clinical trials $(3,4)$.

An earlier report based on data from the National Cancer Institute's Surveillance, Epidemiology, and End Results (SEER) program for the period of 1975-1997 showed AYA cancer patients had the least improvement in 5 -year relative survival 
as compared to both younger and older age groups (5). During that period, survival even worsened for some older AYAs (5). Two subsequent reports using updated SEER data suggest a more complex scenario. For example, since the early 1970s, AYA survival continued to improve for leukemia, kidney cancer, and testicular cancer, but not for cervical cancer, head and neck cancer, or rhabdomyosarcoma (6,7). These reports also recognized the dramatic improvement in survival among AYA males with Kaposi sarcoma (KS) and non-Hodgkin lymphoma, two major cancer types closely associated with human immunodeficiency virus/acquired immunodeficiency syndrome (HIV/AIDS), following the introduction of "highly active antiretroviral therapy" in 1996 (now referred to as "combination antiretroviral therapy" or cART) $(8,9)$. However, the impact of the HIV/AIDS epidemic on overall cancer survival among AYAs has not been fully examined.

Therefore, we undertook this analysis of AYA cancer survival using the most recent SEER data release (10) and adjusting for the historical impact of HIV/AIDS. We hypothesized that previous reports of inferior AYA cancer survival improvement reflected the temporary impact of poor survival among HIV/ AIDS-related cancers (ie, KS and lymphomas) before the availability of antiretroviral therapy. We further hypothesized that AYA cancer survival regained its pre-HIV/AIDS momentum once HIV/AIDS was more effectively controlled with cART.

\section{Methods}

\section{Data Source and Inclusion Criteria}

We used all invasive cancer data (including bladder in situ cases) from the nine SEER registries for 1973-2014 (10) to evaluate the survival of AYA cancer patients (15-39years old) in comparison with children (0-14years) and older adults (40years and older). We grouped age at diagnosis into 5-year age intervals (0-4, $5-9,10-14, \ldots, 55-59, \geq 60)$, as well as broadly aggregated age groups of interest $(0-14,15-39$, and $\geq 40)$. Histology codes from the
International Classification of Disease for Oncology, 3rd Edition were used to identify HIV/AIDS-associated cancers: KS (9140) and lymphomas [9590-9729, 9823, 9827, and 9837; including non-Hodgkin and Hodgkin, as both could be related to HIV/AIDS $(11,12)]$.

\section{Survival Analysis}

We used SEER*Stat software Version 8.3.4 (10) to calculate 5-year relative survival among those diagnosed with cancer during 1973-2009 in this dataset; thus every patient would have had full 5 -year or longer survival time by the end of 2014. Relative survival is defined as the ratio of the observed probability of survival of cancer patients to the expected probability of survival in a comparable set of cancer-free individuals matched by age, race, sex, and year (13). It is a net survival measure representing cancer survival in the absence of other competing causes of death by taking into account the mortality experience in the general population. Two sets of 5-year relative survival were calculated by sex, age, and year of diagnosis for all cancers combined: including and excluding KS and lymphomas, to assess the impact of HIV/AIDS on cancer survival in different population groups.

To evaluate cancer survival improvement over time, we compared the 5-year relative survival by sex and age between the beginning and ending years of the 1973-2009 diagnostic period, as well as between the average 5-year relative survival for the periods of 1973-1977 (before the onset of the HIV/AIDs epidemic in early 1980s) and 2005-2009 (after the HIV/AIDS epidemic was controlled by cART implementation in 1996) to have more statistical stability in the evaluation that is free from the impact of the HIV/AIDS epidemic. The survival improvement was measured by the ratio of the survival probability in the ending year or period divided by the corresponding one from the beginning year or period.

Table 1. Total case counts and 5-year relative survival in males by 5-year age group and diagnostic year, all invasive cancers including and excluding Kaposi sarcoma (KS) and lymphomas, SEER 9 registries

\begin{tabular}{|c|c|c|c|c|c|c|c|c|c|c|c|c|c|}
\hline \multirow[b]{2}{*}{ Diagnostic year } & \multicolumn{13}{|c|}{ 5-year relative survival by age group } \\
\hline & $0-4 y$ & $5-9 y$ & $10-14$ y & $15-19 y$ & $20-24$ y & $25-29 y$ & $30-34$ y & $35-39 y$ & $40-44 y$ & $45-59 y$ & $50-54$ y & $55-59 y$ & $\geq 60 y$ \\
\hline $\begin{array}{l}\text { Including } \mathrm{KS} \text { and } \\
\text { lymphomas, } \mathrm{N}\end{array}$ & 6941 & 3870 & 4196 & 7009 & 11099 & 17107 & 23838 & 31786 & 44636 & 70982 & 117515 & 172337 & 1151099 \\
\hline 1973 & 0.47 & 0.58 & 0.54 & 0.55 & 0.62 & 0.69 & 0.58 & 0.48 & 0.45 & 0.39 & 0.38 & 0.36 & 0.35 \\
\hline 1974 & 0.50 & 0.41 & 0.47 & 0.46 & 0.62 & 0.71 & 0.65 & 0.56 & 0.49 & 0.41 & 0.39 & 0.37 & 0.38 \\
\hline 1975 & 0.53 & 0.52 & 0.48 & 0.56 & 0.67 & 0.67 & 0.67 & 0.59 & 0.50 & 0.43 & 0.40 & 0.40 & 0.39 \\
\hline 1976 & 0.52 & 0.59 & 0.54 & 0.62 & 0.64 & 0.68 & 0.67 & 0.57 & 0.52 & 0.45 & 0.41 & 0.41 & 0.40 \\
\hline 1977 & 0.56 & 0.52 & 0.60 & 0.60 & 0.65 & 0.71 & 0.67 & 0.61 & 0.52 & 0.42 & 0.40 & 0.41 & 0.41 \\
\hline 1978 & 0.66 & 0.60 & 0.56 & 0.59 & 0.65 & 0.73 & 0.67 & 0.58 & 0.50 & 0.42 & 0.40 & 0.41 & 0.41 \\
\hline 1979 & 0.59 & 0.63 & 0.57 & 0.61 & 0.69 & 0.73 & 0.68 & 0.60 & 0.51 & 0.42 & 0.41 & 0.41 & 0.41 \\
\hline 1980 & 0.63 & 0.67 & 0.58 & 0.71 & 0.75 & 0.69 & 0.71 & 0.60 & 0.48 & 0.45 & 0.42 & 0.42 & 0.41 \\
\hline 1981 & 0.64 & 0.57 & 0.59 & 0.61 & 0.77 & 0.74 & 0.72 & 0.60 & 0.53 & 0.47 & 0.42 & 0.44 & 0.43 \\
\hline 1982 & 0.67 & 0.64 & 0.60 & 0.69 & 0.72 & 0.75 & 0.69 & 0.61 & 0.56 & 0.45 & 0.44 & 0.42 & 0.42 \\
\hline 1983 & 0.61 & 0.68 & 0.72 & 0.73 & 0.69 & 0.73 & 0.66 & 0.60 & 0.54 & 0.45 & 0.46 & 0.42 & 0.44 \\
\hline 1984 & 0.64 & 0.70 & 0.65 & 0.63 & 0.72 & 0.65 & 0.58 & 0.56 & 0.52 & 0.46 & 0.44 & 0.43 & 0.44 \\
\hline 1985 & 0.70 & 0.66 & 0.59 & 0.68 & 0.71 & 0.67 & 0.59 & 0.57 & 0.48 & 0.49 & 0.46 & 0.43 & 0.46 \\
\hline 1986 & 0.67 & 0.72 & 0.60 & 0.75 & 0.74 & 0.66 & 0.59 & 0.51 & 0.52 & 0.47 & 0.46 & 0.45 & 0.48 \\
\hline 1987 & 0.77 & 0.68 & 0.59 & 0.71 & 0.74 & 0.63 & 0.56 & 0.52 & 0.50 & 0.50 & 0.49 & 0.48 & 0.50 \\
\hline 1988 & 0.70 & 0.68 & 0.62 & 0.74 & 0.72 & 0.62 & 0.53 & 0.47 & 0.49 & 0.46 & 0.46 & 0.48 & 0.51 \\
\hline 1989 & 0.75 & 0.71 & 0.74 & 0.71 & 0.70 & 0.63 & 0.54 & 0.48 & 0.51 & 0.47 & 0.45 & 0.47 & 0.53 \\
\hline 1990 & 0.76 & 0.84 & 0.76 & 0.73 & 0.77 & 0.64 & 0.52 & 0.50 & 0.51 & 0.49 & 0.51 & 0.50 & 0.56 \\
\hline
\end{tabular}


Table 1. (continued)

5-year relative survival by age group

\begin{tabular}{|c|c|c|c|c|c|c|c|c|c|c|c|c|c|}
\hline Diagnostic year & $0-4 y$ & $5-9 y$ & $10-14$ y & $15-19 y$ & $20-24$ y & $25-29 y$ & $30-34$ y & $35-39 y$ & $40-44$ y & $45-59 y$ & $50-54$ y & $55-59 y$ & $\geq 60 y$ \\
\hline 1991 & 0.73 & 0.75 & 0.74 & 0.79 & 0.74 & 0.59 & 0.52 & 0.49 & 0.51 & 0.50 & 0.51 & 0.54 & 0.61 \\
\hline 1992 & 0.77 & 0.78 & 0.81 & 0.72 & 0.76 & 0.61 & 0.53 & 0.51 & 0.51 & 0.51 & 0.56 & 0.59 & 0.65 \\
\hline 1993 & 0.73 & 0.74 & 0.74 & 0.72 & 0.79 & 0.63 & 0.56 & 0.53 & 0.52 & 0.55 & 0.58 & 0.61 & 0.63 \\
\hline 1994 & 0.81 & 0.81 & 0.73 & 0.73 & 0.75 & 0.70 & 0.55 & 0.59 & 0.54 & 0.56 & 0.60 & 0.60 & 0.61 \\
\hline 1995 & 0.75 & 0.80 & 0.77 & 0.75 & 0.75 & 0.72 & 0.63 & 0.57 & 0.59 & 0.58 & 0.59 & 0.62 & 0.61 \\
\hline 1996 & 0.83 & 0.79 & 0.76 & 0.81 & 0.74 & 0.77 & 0.70 & 0.66 & 0.61 & 0.62 & 0.63 & 0.63 & 0.61 \\
\hline 1997 & 0.78 & 0.84 & 0.75 & 0.76 & 0.82 & 0.78 & 0.73 & 0.70 & 0.60 & 0.62 & 0.64 & 0.63 & 0.62 \\
\hline 1998 & 0.78 & 0.79 & 0.80 & 0.75 & 0.78 & 0.84 & 0.75 & 0.73 & 0.65 & 0.63 & 0.65 & 0.66 & 0.62 \\
\hline 1999 & 0.80 & 0.76 & 0.75 & 0.73 & 0.83 & 0.80 & 0.77 & 0.71 & 0.66 & 0.61 & 0.65 & 0.68 & 0.64 \\
\hline 2000 & 0.81 & 0.80 & 0.78 & 0.72 & 0.83 & 0.82 & 0.78 & 0.72 & 0.67 & 0.64 & 0.67 & 0.70 & 0.65 \\
\hline 2001 & 0.84 & 0.80 & 0.76 & 0.79 & 0.82 & 0.79 & 0.79 & 0.71 & 0.71 & 0.66 & 0.68 & 0.70 & 0.66 \\
\hline 2002 & 0.84 & 0.74 & 0.77 & 0.76 & 0.80 & 0.81 & 0.81 & 0.77 & 0.70 & 0.66 & 0.70 & 0.71 & 0.66 \\
\hline 2003 & 0.80 & 0.81 & 0.86 & 0.83 & 0.81 & 0.82 & 0.80 & 0.74 & 0.72 & 0.67 & 0.68 & 0.70 & 0.65 \\
\hline 2004 & 0.84 & 0.80 & 0.83 & 0.79 & 0.84 & 0.83 & 0.80 & 0.76 & 0.73 & 0.67 & 0.69 & 0.71 & 0.66 \\
\hline 2005 & 0.83 & 0.83 & 0.78 & 0.85 & 0.84 & 0.82 & 0.83 & 0.79 & 0.74 & 0.68 & 0.69 & 0.70 & 0.66 \\
\hline 2006 & 0.83 & 0.87 & 0.84 & 0.81 & 0.83 & 0.82 & 0.80 & 0.76 & 0.75 & 0.70 & 0.70 & 0.73 & 0.68 \\
\hline 2007 & 0.83 & 0.87 & 0.82 & 0.81 & 0.81 & 0.83 & 0.83 & 0.80 & 0.74 & 0.70 & 0.71 & 0.73 & 0.68 \\
\hline 2008 & 0.86 & 0.84 & 0.81 & 0.82 & 0.85 & 0.84 & 0.84 & 0.80 & 0.74 & 0.73 & 0.71 & 0.72 & 0.67 \\
\hline 2009 & 0.82 & 0.77 & 0.86 & 0.84 & 0.84 & 0.84 & 0.81 & 0.77 & 0.76 & 0.72 & 0.72 & 0.72 & 0.67 \\
\hline $\begin{array}{c}\text { Survival ratio } \\
2009: 1973\end{array}$ & 1.74 & 1.33 & 1.59 & 1.53 & 1.35 & 1.22 & 1.40 & 1.60 & 1.69 & 1.85 & 1.89 & 2.00 & 1.91 \\
\hline $\begin{array}{l}\text { Excluding KS and } \\
\text { lymphomas, } \mathrm{N}\end{array}$ & 6679 & 3258 & 3229 & 5200 & 8285 & 12486 & 16982 & 23910 & 37229 & 64016 & 110274 & 164757 & 1112041 \\
\hline 1973 & 0.46 & 0.57 & 0.44 & 0.44 & 0.56 & 0.70 & 0.57 & 0.47 & 0.45 & 0.38 & 0.37 & 0.36 & 0.35 \\
\hline 1974 & 0.48 & 0.41 & 0.44 & 0.42 & 0.59 & 0.69 & 0.63 & 0.53 & 0.48 & 0.40 & 0.39 & 0.36 & 0.38 \\
\hline 1975 & 0.55 & 0.53 & 0.43 & 0.50 & 0.61 & 0.67 & 0.64 & 0.57 & 0.48 & 0.41 & 0.39 & 0.39 & 0.39 \\
\hline 1976 & 0.53 & 0.57 & 0.52 & 0.54 & 0.62 & 0.66 & 0.65 & 0.55 & 0.52 & 0.44 & 0.40 & 0.40 & 0.40 \\
\hline 1977 & 0.57 & 0.52 & 0.60 & 0.56 & 0.64 & 0.72 & 0.67 & 0.59 & 0.51 & 0.41 & 0.39 & 0.41 & 0.41 \\
\hline 1978 & 0.68 & 0.62 & 0.52 & 0.53 & 0.63 & 0.72 & 0.65 & 0.57 & 0.48 & 0.41 & 0.39 & 0.40 & 0.41 \\
\hline 1979 & 0.60 & 0.57 & 0.50 & 0.55 & 0.68 & 0.72 & 0.65 & 0.59 & 0.50 & 0.41 & 0.41 & 0.41 & 0.41 \\
\hline 1980 & 0.63 & 0.62 & 0.52 & 0.69 & 0.71 & 0.67 & 0.71 & 0.58 & 0.48 & 0.44 & 0.41 & 0.41 & 0.42 \\
\hline 1981 & 0.64 & 0.57 & 0.53 & 0.59 & 0.74 & 0.73 & 0.72 & 0.60 & 0.52 & 0.46 & 0.41 & 0.43 & 0.43 \\
\hline 1982 & 0.68 & 0.63 & 0.58 & 0.63 & 0.73 & 0.75 & 0.70 & 0.61 & 0.55 & 0.44 & 0.43 & 0.41 & 0.42 \\
\hline 1983 & 0.60 & 0.60 & 0.69 & 0.71 & 0.68 & 0.75 & 0.70 & 0.63 & 0.54 & 0.45 & 0.45 & 0.42 & 0.44 \\
\hline 1984 & 0.64 & 0.64 & 0.59 & 0.57 & 0.72 & 0.70 & 0.67 & 0.58 & 0.53 & 0.46 & 0.43 & 0.42 & 0.44 \\
\hline 1985 & 0.71 & 0.61 & 0.56 & 0.66 & 0.73 & 0.79 & 0.71 & 0.67 & 0.49 & 0.49 & 0.45 & 0.43 & 0.46 \\
\hline 1986 & 0.66 & 0.72 & 0.52 & 0.72 & 0.77 & 0.81 & 0.76 & 0.64 & 0.57 & 0.47 & 0.45 & 0.44 & 0.48 \\
\hline 1987 & 0.77 & 0.65 & 0.54 & 0.64 & 0.78 & 0.78 & 0.76 & 0.66 & 0.56 & 0.52 & 0.48 & 0.47 & 0.50 \\
\hline 1988 & 0.70 & 0.68 & 0.55 & 0.72 & 0.73 & 0.80 & 0.74 & 0.64 & 0.56 & 0.46 & 0.45 & 0.48 & 0.52 \\
\hline 1989 & 0.75 & 0.69 & 0.74 & 0.69 & 0.73 & 0.82 & 0.77 & 0.66 & 0.59 & 0.49 & 0.45 & 0.47 & 0.53 \\
\hline 1990 & 0.75 & 0.83 & 0.71 & 0.67 & 0.81 & 0.81 & 0.75 & 0.67 & 0.59 & 0.52 & 0.51 & 0.50 & 0.56 \\
\hline 1991 & 0.72 & 0.74 & 0.71 & 0.77 & 0.79 & 0.77 & 0.75 & 0.66 & 0.59 & 0.53 & 0.52 & 0.55 & 0.62 \\
\hline 1992 & 0.76 & 0.77 & 0.81 & 0.70 & 0.76 & 0.79 & 0.75 & 0.67 & 0.59 & 0.53 & 0.56 & 0.60 & 0.65 \\
\hline 1993 & 0.73 & 0.72 & 0.71 & 0.69 & 0.83 & 0.82 & 0.77 & 0.69 & 0.59 & 0.57 & 0.58 & 0.61 & 0.63 \\
\hline 1994 & 0.81 & 0.80 & 0.71 & 0.69 & 0.77 & 0.85 & 0.73 & 0.71 & 0.61 & 0.59 & 0.59 & 0.60 & 0.61 \\
\hline 1995 & 0.75 & 0.77 & 0.75 & 0.71 & 0.76 & 0.83 & 0.78 & 0.68 & 0.63 & 0.59 & 0.59 & 0.62 & 0.61 \\
\hline 1996 & 0.83 & 0.78 & 0.72 & 0.77 & 0.78 & 0.83 & 0.79 & 0.72 & 0.63 & 0.62 & 0.63 & 0.63 & 0.62 \\
\hline 1997 & 0.77 & 0.83 & 0.72 & 0.73 & 0.79 & 0.77 & 0.77 & 0.70 & 0.60 & 0.62 & 0.64 & 0.63 & 0.63 \\
\hline 1998 & 0.78 & 0.78 & 0.77 & 0.73 & 0.79 & 0.85 & 0.77 & 0.75 & 0.65 & 0.62 & 0.65 & 0.66 & 0.62 \\
\hline 1999 & 0.80 & 0.73 & 0.70 & 0.68 & 0.83 & 0.80 & 0.81 & 0.72 & 0.66 & 0.60 & 0.65 & 0.68 & 0.65 \\
\hline 2000 & 0.81 & 0.78 & 0.71 & 0.72 & 0.82 & 0.82 & 0.79 & 0.74 & 0.66 & 0.64 & 0.67 & 0.69 & 0.65 \\
\hline 2001 & 0.84 & 0.79 & 0.72 & 0.77 & 0.81 & 0.82 & 0.80 & 0.72 & 0.71 & 0.65 & 0.68 & 0.71 & 0.66 \\
\hline 2002 & 0.83 & 0.71 & 0.75 & 0.71 & 0.79 & 0.80 & 0.81 & 0.78 & 0.69 & 0.65 & 0.69 & 0.71 & 0.66 \\
\hline 2003 & 0.80 & 0.79 & 0.84 & 0.79 & 0.79 & 0.83 & 0.80 & 0.75 & 0.71 & 0.66 & 0.68 & 0.70 & 0.65 \\
\hline 2004 & 0.84 & 0.78 & 0.83 & 0.79 & 0.82 & 0.82 & 0.80 & 0.77 & 0.73 & 0.67 & 0.69 & 0.71 & 0.66 \\
\hline 2005 & 0.83 & 0.81 & 0.77 & 0.82 & 0.81 & 0.84 & 0.83 & 0.79 & 0.72 & 0.67 & 0.69 & 0.70 & 0.66 \\
\hline 2006 & 0.82 & 0.86 & 0.80 & 0.80 & 0.82 & 0.83 & 0.79 & 0.76 & 0.74 & 0.69 & 0.69 & 0.73 & 0.68 \\
\hline 2007 & 0.82 & 0.86 & 0.79 & 0.78 & 0.80 & 0.81 & 0.83 & 0.79 & 0.74 & 0.69 & 0.70 & 0.73 & 0.69 \\
\hline 2008 & 0.87 & 0.85 & 0.77 & 0.80 & 0.83 & 0.83 & 0.84 & 0.79 & 0.74 & 0.72 & 0.70 & 0.72 & 0.67 \\
\hline 2009 & 0.82 & 0.74 & 0.85 & 0.82 & 0.84 & 0.84 & 0.82 & 0.77 & 0.76 & 0.71 & 0.72 & 0.71 & 0.68 \\
\hline $\begin{array}{c}\text { Survival ratio } \\
2009: 1973\end{array}$ & 1.78 & 1.30 & 1.93 & 1.86 & 1.50 & 1.20 & 1.44 & 1.64 & 1.69 & 1.87 & 1.95 & 1.97 & 1.94 \\
\hline
\end{tabular}


Table 2. Total case counts and 5-year relative survival in females by 5-year age group and diagnostic year, all invasive cancers including and excluding Kaposi sarcoma (KS) and lymphomas, SEER 9 registries

5-year relative survival by age group

\begin{tabular}{|c|c|c|c|c|c|c|c|c|c|c|c|c|c|}
\hline Diagnostic year & $0-4 y$ & $5-9 y$ & $10-14 y$ & $15-19 y$ & $20-24$ y & $25-29 y$ & $30-34 y$ & $35-39 y$ & $40-44$ y & $45-59 y$ & $50-54$ y & $55-59 y$ & $\geq 60 y$ \\
\hline $\begin{array}{l}\text { Including KS and } \\
\text { lymphomas, } N\end{array}$ & 5929 & 3017 & 3693 & 6324 & 11436 & 20606 & 33246 & 51479 & 77774 & 107866 & 133119 & 154820 & 937997 \\
\hline 1973 & 0.44 & 0.47 & 0.61 & 0.71 & 0.76 & 0.77 & 0.75 & 0.70 & 0.64 & 0.65 & 0.62 & 0.58 & 0.45 \\
\hline 1974 & 0.65 & 0.55 & 0.64 & 0.70 & 0.78 & 0.77 & 0.72 & 0.73 & 0.66 & 0.67 & 0.63 & 0.59 & 0.48 \\
\hline 1975 & 0.60 & 0.64 & 0.60 & 0.72 & 0.76 & 0.79 & 0.73 & 0.71 & 0.70 & 0.66 & 0.64 & 0.60 & 0.49 \\
\hline 1976 & 0.64 & 0.62 & 0.59 & 0.77 & 0.77 & 0.79 & 0.77 & 0.72 & 0.69 & 0.67 & 0.64 & 0.60 & 0.48 \\
\hline 1977 & 0.59 & 0.63 & 0.69 & 0.79 & 0.81 & 0.80 & 0.77 & 0.73 & 0.71 & 0.65 & 0.63 & 0.59 & 0.48 \\
\hline 1978 & 0.67 & 0.58 & 0.71 & 0.76 & 0.81 & 0.82 & 0.80 & 0.70 & 0.70 & 0.65 & 0.64 & 0.57 & 0.49 \\
\hline 1979 & 0.69 & 0.63 & 0.57 & 0.79 & 0.77 & 0.78 & 0.77 & 0.70 & 0.70 & 0.65 & 0.62 & 0.56 & 0.48 \\
\hline 1980 & 0.59 & 0.66 & 0.66 & 0.76 & 0.86 & 0.83 & 0.74 & 0.73 & 0.71 & 0.65 & 0.60 & 0.58 & 0.48 \\
\hline 1981 & 0.69 & 0.75 & 0.63 & 0.75 & 0.79 & 0.82 & 0.76 & 0.73 & 0.71 & 0.68 & 0.60 & 0.59 & 0.48 \\
\hline 1982 & 0.72 & 0.75 & 0.72 & 0.73 & 0.80 & 0.82 & 0.76 & 0.75 & 0.70 & 0.68 & 0.61 & 0.58 & 0.48 \\
\hline 1983 & 0.71 & 0.69 & 0.72 & 0.76 & 0.81 & 0.81 & 0.75 & 0.75 & 0.70 & 0.68 & 0.63 & 0.58 & 0.49 \\
\hline 1984 & 0.68 & 0.67 & 0.61 & 0.79 & 0.83 & 0.81 & 0.77 & 0.75 & 0.71 & 0.69 & 0.63 & 0.59 & 0.50 \\
\hline 1985 & 0.72 & 0.75 & 0.74 & 0.85 & 0.87 & 0.83 & 0.76 & 0.72 & 0.74 & 0.68 & 0.66 & 0.60 & 0.51 \\
\hline 1986 & 0.72 & 0.72 & 0.71 & 0.84 & 0.88 & 0.83 & 0.79 & 0.77 & 0.71 & 0.70 & 0.67 & 0.60 & 0.52 \\
\hline 1987 & 0.73 & 0.72 & 0.69 & 0.86 & 0.83 & 0.80 & 0.80 & 0.77 & 0.75 & 0.71 & 0.65 & 0.61 & 0.53 \\
\hline 1988 & 0.74 & 0.78 & 0.87 & 0.83 & 0.86 & 0.81 & 0.80 & 0.77 & 0.76 & 0.72 & 0.67 & 0.62 & 0.54 \\
\hline 1989 & 0.66 & 0.72 & 0.76 & 0.80 & 0.87 & 0.84 & 0.78 & 0.76 & 0.76 & 0.72 & 0.68 & 0.63 & 0.53 \\
\hline 1990 & 0.73 & 0.62 & 0.68 & 0.81 & 0.87 & 0.83 & 0.82 & 0.75 & 0.77 & 0.74 & 0.67 & 0.62 & 0.54 \\
\hline 1991 & 0.78 & 0.75 & 0.78 & 0.81 & 0.85 & 0.83 & 0.79 & 0.79 & 0.77 & 0.75 & 0.69 & 0.63 & 0.54 \\
\hline 1992 & 0.80 & 0.68 & 0.79 & 0.79 & 0.83 & 0.82 & 0.81 & 0.80 & 0.78 & 0.74 & 0.69 & 0.65 & 0.54 \\
\hline 1993 & 0.80 & 0.80 & 0.80 & 0.82 & 0.85 & 0.84 & 0.81 & 0.81 & 0.78 & 0.74 & 0.70 & 0.65 & 0.54 \\
\hline 1994 & 0.74 & 0.86 & 0.79 & 0.83 & 0.85 & 0.82 & 0.82 & 0.79 & 0.78 & 0.75 & 0.71 & 0.66 & 0.54 \\
\hline 1995 & 0.74 & 0.85 & 0.81 & 0.86 & 0.85 & 0.83 & 0.80 & 0.80 & 0.79 & 0.77 & 0.73 & 0.69 & 0.54 \\
\hline 1996 & 0.80 & 0.78 & 0.84 & 0.89 & 0.89 & 0.87 & 0.82 & 0.81 & 0.80 & 0.77 & 0.72 & 0.68 & 0.55 \\
\hline 1997 & 0.79 & 0.75 & 0.85 & 0.80 & 0.88 & 0.87 & 0.81 & 0.82 & 0.79 & 0.78 & 0.75 & 0.69 & 0.55 \\
\hline 1998 & 0.78 & 0.73 & 0.79 & 0.86 & 0.84 & 0.85 & 0.82 & 0.81 & 0.81 & 0.77 & 0.75 & 0.71 & 0.56 \\
\hline 1999 & 0.82 & 0.81 & 0.83 & 0.88 & 0.86 & 0.86 & 0.86 & 0.82 & 0.81 & 0.79 & 0.76 & 0.71 & 0.56 \\
\hline 2000 & 0.80 & 0.82 & 0.83 & 0.80 & 0.85 & 0.87 & 0.84 & 0.83 & 0.81 & 0.81 & 0.76 & 0.72 & 0.57 \\
\hline 2001 & 0.80 & 0.89 & 0.84 & 0.84 & 0.90 & 0.87 & 0.84 & 0.83 & 0.81 & 0.78 & 0.77 & 0.72 & 0.58 \\
\hline 2002 & 0.84 & 0.85 & 0.81 & 0.87 & 0.83 & 0.87 & 0.87 & 0.84 & 0.82 & 0.80 & 0.76 & 0.73 & 0.58 \\
\hline 2003 & 0.87 & 0.83 & 0.89 & 0.86 & 0.88 & 0.90 & 0.86 & 0.86 & 0.83 & 0.80 & 0.75 & 0.73 & 0.57 \\
\hline 2004 & 0.85 & 0.77 & 0.85 & 0.83 & 0.90 & 0.89 & 0.86 & 0.86 & 0.83 & 0.79 & 0.77 & 0.73 & 0.57 \\
\hline 2005 & 0.81 & 0.81 & 0.86 & 0.88 & 0.85 & 0.87 & 0.85 & 0.86 & 0.84 & 0.81 & 0.77 & 0.74 & 0.58 \\
\hline 2006 & 0.90 & 0.86 & 0.86 & 0.86 & 0.89 & 0.89 & 0.88 & 0.86 & 0.84 & 0.82 & 0.78 & 0.75 & 0.58 \\
\hline 2007 & 0.85 & 0.79 & 0.84 & 0.86 & 0.89 & 0.88 & 0.90 & 0.86 & 0.85 & 0.82 & 0.78 & 0.74 & 0.59 \\
\hline 2008 & 0.82 & 0.74 & 0.91 & 0.89 & 0.91 & 0.91 & 0.88 & 0.88 & 0.85 & 0.82 & 0.79 & 0.74 & 0.59 \\
\hline 2009 & 0.83 & 0.82 & 0.85 & 0.91 & 0.91 & 0.88 & 0.88 & 0.85 & 0.85 & 0.82 & 0.78 & 0.75 & 0.60 \\
\hline $\begin{array}{c}\text { Survival ratio } \\
2009: 1973\end{array}$ & 1.89 & 1.74 & 1.39 & 1.28 & 1.20 & 1.14 & 1.17 & 1.21 & 1.33 & 1.26 & 1.26 & 1.29 & 1.33 \\
\hline $\begin{array}{l}\text { Excluding } \mathrm{KS} \text { and } \\
\text { lymphomas, } \mathrm{N}\end{array}$ & 5792 & 2786 & 3059 & 4764 & 9209 & 18088 & 30816 & 49001 & 74886 & 104336 & 128671 & 149470 & 898382 \\
\hline 1973 & 0.44 & 0.47 & 0.57 & 0.67 & 0.76 & 0.77 & 0.75 & 0.70 & 0.64 & 0.65 & 0.62 & 0.58 & 0.46 \\
\hline 1974 & 0.63 & 0.55 & 0.63 & 0.65 & 0.80 & 0.77 & 0.72 & 0.73 & 0.66 & 0.67 & 0.63 & 0.60 & 0.49 \\
\hline 1975 & 0.60 & 0.66 & 0.58 & 0.69 & 0.76 & 0.79 & 0.72 & 0.71 & 0.70 & 0.66 & 0.64 & 0.61 & 0.49 \\
\hline 1976 & 0.65 & 0.60 & 0.57 & 0.73 & 0.78 & 0.79 & 0.78 & 0.72 & 0.69 & 0.67 & 0.64 & 0.60 & 0.49 \\
\hline 1977 & 0.59 & 0.62 & 0.66 & 0.75 & 0.80 & 0.79 & 0.77 & 0.73 & 0.71 & 0.64 & 0.63 & 0.59 & 0.48 \\
\hline 1978 & 0.67 & 0.58 & 0.67 & 0.72 & 0.80 & 0.82 & 0.80 & 0.70 & 0.70 & 0.65 & 0.64 & 0.57 & 0.49 \\
\hline 1979 & 0.70 & 0.63 & 0.56 & 0.75 & 0.77 & 0.78 & 0.78 & 0.69 & 0.70 & 0.64 & 0.62 & 0.56 & 0.48 \\
\hline 1980 & 0.59 & 0.67 & 0.64 & 0.75 & 0.87 & 0.84 & 0.73 & 0.72 & 0.71 & 0.65 & 0.60 & 0.58 & 0.48 \\
\hline 1981 & 0.69 & 0.73 & 0.59 & 0.72 & 0.79 & 0.82 & 0.76 & 0.73 & 0.71 & 0.68 & 0.60 & 0.59 & 0.48 \\
\hline 1982 & 0.73 & 0.76 & 0.70 & 0.70 & 0.81 & 0.82 & 0.76 & 0.75 & 0.69 & 0.69 & 0.61 & 0.58 & 0.48 \\
\hline 1983 & 0.72 & 0.70 & 0.70 & 0.75 & 0.80 & 0.81 & 0.74 & 0.75 & 0.70 & 0.68 & 0.63 & 0.58 & 0.49 \\
\hline 1984 & 0.69 & 0.65 & 0.61 & 0.75 & 0.81 & 0.80 & 0.77 & 0.74 & 0.71 & 0.68 & 0.63 & 0.59 & 0.51 \\
\hline 1985 & 0.72 & 0.77 & 0.71 & 0.86 & 0.85 & 0.83 & 0.76 & 0.72 & 0.75 & 0.68 & 0.66 & 0.60 & 0.52 \\
\hline 1986 & 0.71 & 0.73 & 0.67 & 0.81 & 0.88 & 0.82 & 0.80 & 0.77 & 0.71 & 0.70 & 0.67 & 0.60 & 0.53 \\
\hline 1987 & 0.72 & 0.72 & 0.67 & 0.86 & 0.81 & 0.81 & 0.80 & 0.77 & 0.75 & 0.71 & 0.65 & 0.61 & 0.53 \\
\hline 1988 & 0.75 & 0.76 & 0.88 & 0.83 & 0.84 & 0.81 & 0.81 & 0.77 & 0.76 & 0.72 & 0.67 & 0.62 & 0.54 \\
\hline 1989 & 0.65 & 0.71 & 0.70 & 0.78 & 0.86 & 0.84 & 0.78 & 0.77 & 0.76 & 0.72 & 0.68 & 0.62 & 0.53 \\
\hline 1990 & 0.74 & 0.61 & 0.65 & 0.81 & 0.86 & 0.82 & 0.81 & 0.75 & 0.77 & 0.74 & 0.67 & 0.62 & 0.55 \\
\hline 1991 & 0.79 & 0.75 & 0.76 & 0.79 & 0.85 & 0.83 & 0.80 & 0.79 & 0.77 & 0.75 & 0.69 & 0.63 & 0.54 \\
\hline 1992 & 0.80 & 0.67 & 0.78 & 0.77 & 0.83 & 0.82 & 0.81 & 0.80 & 0.78 & 0.74 & 0.69 & 0.65 & 0.54 \\
\hline 1993 & 0.80 & 0.78 & 0.77 & 0.81 & 0.84 & 0.84 & 0.81 & 0.81 & 0.78 & 0.74 & 0.70 & 0.65 & 0.54 \\
\hline 1994 & 0.74 & 0.85 & 0.77 & 0.79 & 0.84 & 0.81 & 0.82 & 0.79 & 0.78 & 0.76 & 0.71 & 0.66 & 0.55 \\
\hline 1995 & 0.75 & 0.84 & 0.81 & 0.85 & 0.86 & 0.83 & 0.81 & 0.79 & 0.79 & 0.77 & 0.73 & 0.69 & 0.54 \\
\hline 1996 & 0.81 & 0.78 & 0.81 & 0.87 & 0.89 & 0.86 & 0.83 & 0.81 & 0.80 & 0.77 & 0.72 & 0.68 & 0.55 \\
\hline
\end{tabular}


Table 2. (continued)

\begin{tabular}{|c|c|c|c|c|c|c|c|c|c|c|c|c|c|}
\hline \multirow[b]{2}{*}{ Diagnostic year } & \multicolumn{13}{|c|}{ 5-year relative survival by age group } \\
\hline & $0-4 y$ & $5-9 y$ & $10-14$ y & $15-19 y$ & $20-24$ y & $25-29 y$ & $30-34$ y & $35-39 y$ & $40-44$ y & $45-59 y$ & $50-54$ y & $55-59$ y & $\geq 60 y$ \\
\hline 1997 & 0.79 & 0.74 & 0.82 & 0.77 & 0.87 & 0.87 & 0.82 & 0.82 & 0.79 & 0.77 & 0.75 & 0.69 & 0.55 \\
\hline 1998 & 0.78 & 0.73 & 0.78 & 0.82 & 0.82 & 0.86 & 0.83 & 0.81 & 0.81 & 0.77 & 0.75 & 0.71 & 0.56 \\
\hline 1999 & 0.83 & 0.81 & 0.81 & 0.85 & 0.87 & 0.86 & 0.86 & 0.82 & 0.81 & 0.79 & 0.76 & 0.71 & 0.56 \\
\hline 2000 & 0.80 & 0.80 & 0.80 & 0.77 & 0.83 & 0.88 & 0.84 & 0.82 & 0.81 & 0.81 & 0.76 & 0.72 & 0.57 \\
\hline 2001 & 0.80 & 0.87 & 0.84 & 0.83 & 0.90 & 0.87 & 0.84 & 0.83 & 0.81 & 0.78 & 0.77 & 0.72 & 0.57 \\
\hline 2002 & 0.84 & 0.83 & 0.81 & 0.86 & 0.83 & 0.88 & 0.86 & 0.84 & 0.82 & 0.79 & 0.76 & 0.72 & 0.57 \\
\hline 2003 & 0.86 & 0.83 & 0.87 & 0.85 & 0.87 & 0.89 & 0.86 & 0.85 & 0.83 & 0.80 & 0.75 & 0.72 & 0.56 \\
\hline 2004 & 0.85 & 0.78 & 0.85 & 0.78 & 0.89 & 0.90 & 0.86 & 0.85 & 0.83 & 0.79 & 0.76 & 0.72 & 0.57 \\
\hline 2005 & 0.81 & 0.81 & 0.85 & 0.88 & 0.84 & 0.86 & 0.84 & 0.86 & 0.83 & 0.81 & 0.77 & 0.74 & 0.57 \\
\hline 2006 & 0.90 & 0.84 & 0.85 & 0.86 & 0.89 & 0.88 & 0.87 & 0.85 & 0.84 & 0.82 & 0.78 & 0.74 & 0.57 \\
\hline 2007 & 0.84 & 0.78 & 0.83 & 0.83 & 0.87 & 0.87 & 0.89 & 0.86 & 0.84 & 0.82 & 0.78 & 0.74 & 0.58 \\
\hline 2008 & 0.82 & 0.73 & 0.90 & 0.86 & 0.91 & 0.91 & 0.88 & 0.87 & 0.86 & 0.82 & 0.78 & 0.74 & 0.59 \\
\hline 2009 & 0.83 & 0.82 & 0.82 & 0.91 & 0.90 & 0.87 & 0.88 & 0.85 & 0.85 & 0.81 & 0.78 & 0.74 & 0.60 \\
\hline $\begin{array}{c}\text { Survival ratio } \\
2009: 1973\end{array}$ & 1.89 & 1.74 & 1.44 & 1.36 & 1.18 & 1.13 & 1.17 & 1.21 & 1.33 & 1.25 & 1.26 & 1.28 & 1.30 \\
\hline
\end{tabular}
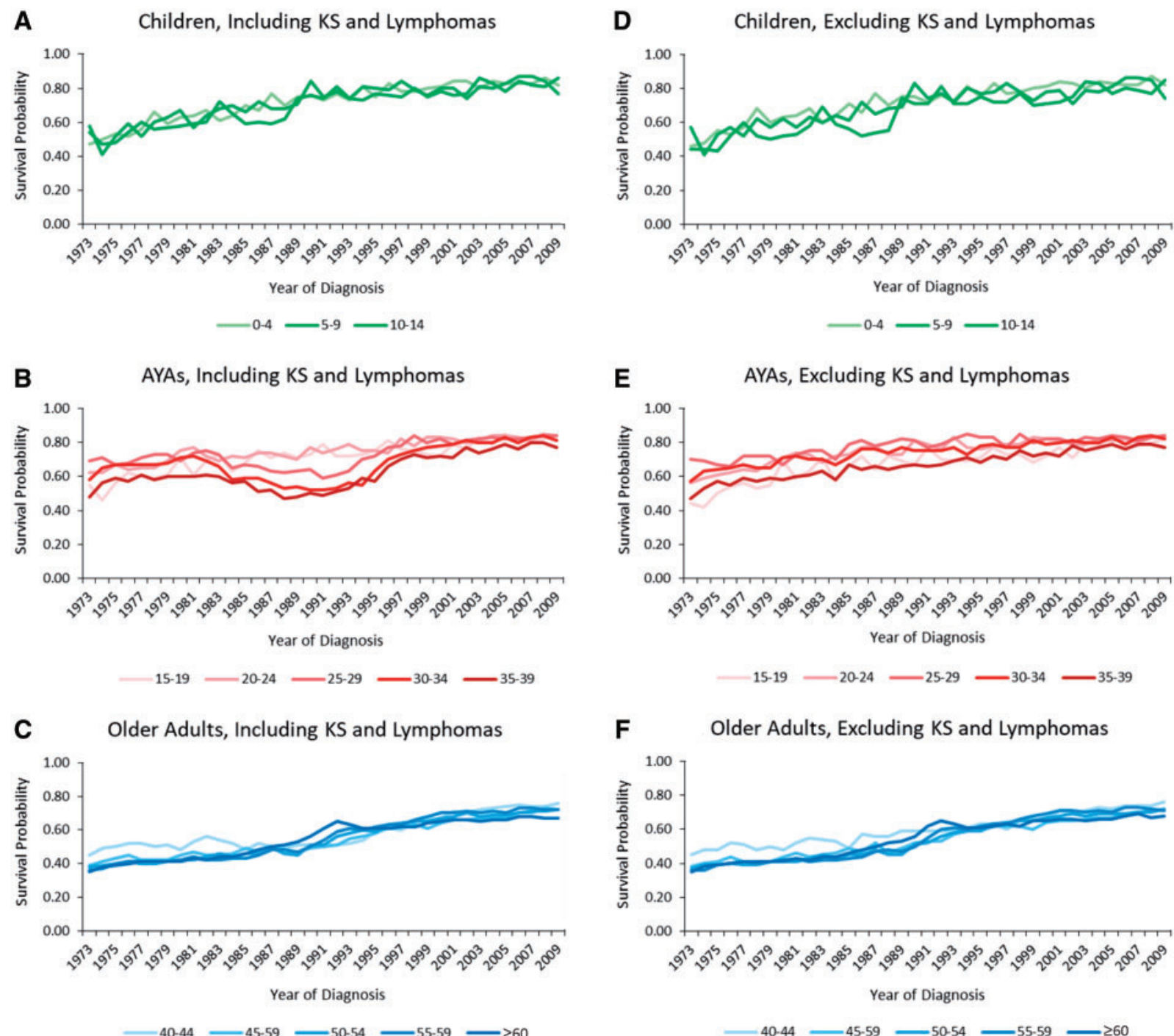

Figure 1. Time trends of 5-year relative survival in males by 5-year age group. Data were from the SEER 9 registries, 1973-2009. Survival probability of all invasive cancers including Kaposi sarcoma (KS) and lymphomas are shown in (A) children age 0-14 years, (B) adolescents and young adults (AYAs) age 15-39 years, and (C) older adults age 40 to $\geq 60$ years, respectively. Survival probability of all invasive cancers excluding KS and lymphomas are shown in (D) children age 0-14 years, (E) AYAs age $15-39$ years, and (F) older adults age 40 to $\geq 60$ years, respectively. 

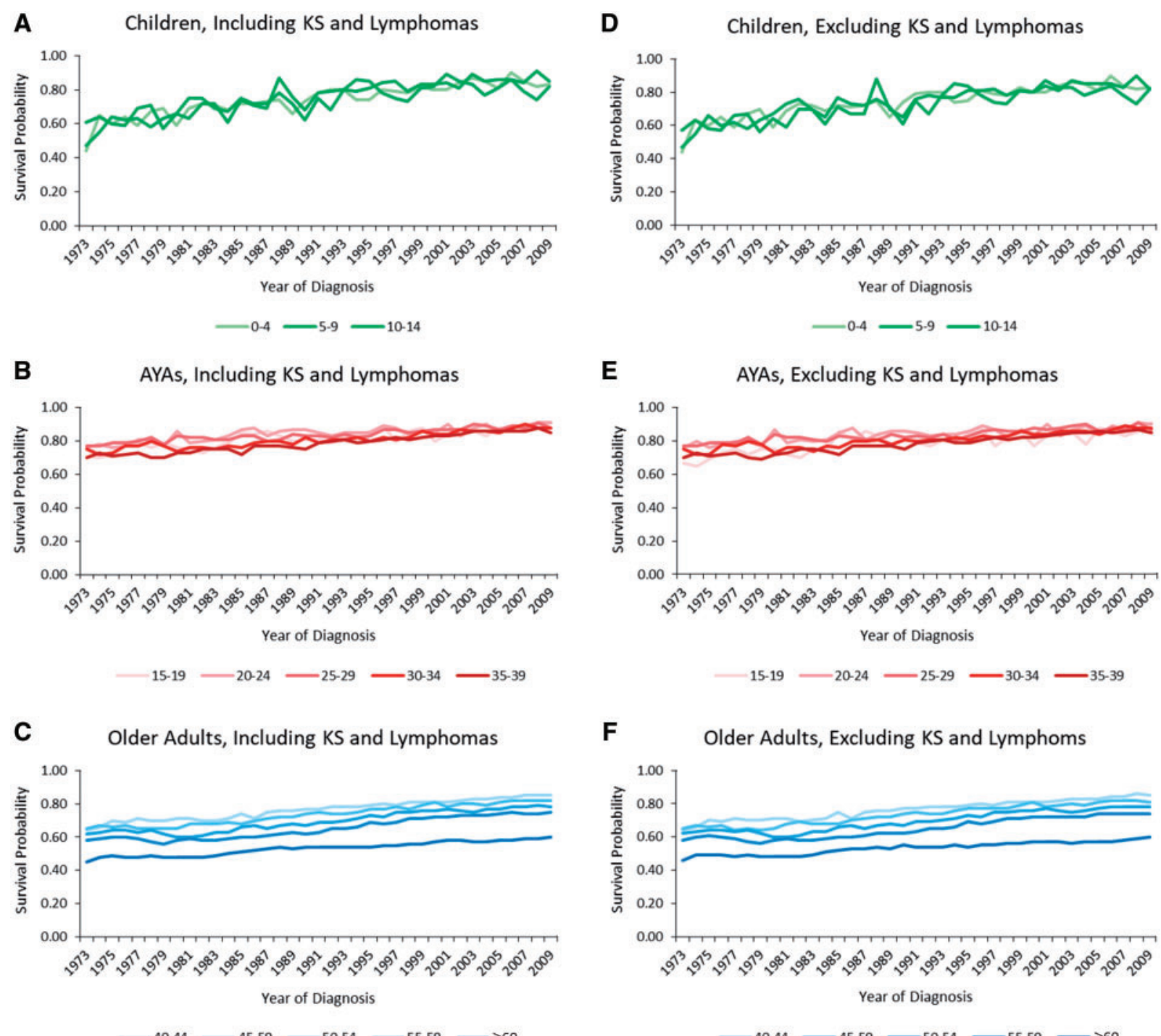

Figure 2. Time trends of 5-year relative survival for females by 5 -year age group. Data were from the SEER 9 registries, 1973-2009. Survival probability of all invasive cancers including Kaposi sarcoma (KS) and lymphomas are shown in (A) children age 0-14 years, (B) adolescents and young adults (AYAs) age 15-39 years, and (C) older adults age 40 to $\geq 60$ years, respectively. Survival probability of all invasive cancers excluding KS and lymphomas are shown in (D) children age $0-14$ years, (E) AYAs age 15-39years, and (F) older adults age 40 to $\geq 60$ years, respectively.

\section{Results}

A total of 3209721 invasive cancer cases were included in the study, with 27646 children, 213930 AYAs, and 2968145 older adults. Exclusion of $\mathrm{KS}$ and lymphoma resulted in case count reduction 24803 in children, 178741 in AYAs, and 2844062 in older adults.

AYAs displayed better overall cancer survival than children or older adults in the 1970s, based on both the 5-year age grouping (Tables 1 and 2 and Figures 1 and 2) and the three broad age groups (Table 3 and Figure 3). For example, during 1973-1979, the overall 5-year relative survival among male AYAs ranged from 0.58 to 0.67 as compared to $0.47-0.61$ for male children and 0.36-0.42 for male older adults; among female AYAs the numbers were $0.73-0.77$ as compared with $0.51-0.65$ for female children and 0.52-0.55 for female older adults (Table 3). The 5-year relative survival for all cancers combined including KS and lymphomas has been improving since the early 1970 s across all female age groups (Table 2 and Figure 2, A-C; Table 3 and Figure 3, B) and for male children and older males (Table 1 and Figure 1, A and C; Table 3 and Figure 3, A). For male AYAs there was a deficit in 5-year relative survival among those 25-39years old (Table 1 and Figure 1, B; Table 3 and Figure 3, A) during 1983-1997, a period that corresponds to the initial uncontrolled HIV/AIDS epidemic, prior to the availability of effective cART. After excluding KS and lymphomas, this male AYA survival deficit disappeared (Table 1 and Figure 1, E; Table 3 and Figure 3, C), which demonstrates the negative impact of uncontrolled HIV/AIDS on the overall cancer survival among male AYAs during this time period. Inclusion or exclusion of $\mathrm{KS}$ and lymphomas did not result in noticeable changes in the survival probability for males of either younger or older age groups (Figure 1, A, D, C, F; Figure 3, $A$ and $C$ ), or for females of any age group (Figures 2, A-F; Figure 3, B and D). Apart from the dip in survival probabilities 
Table 3. Five-year relative survival by sex, broad age group, and diagnostic year, all invasive cancers including and excluding Kaposi sarcoma (KS) and lymphomas, SEER 9 registries

\begin{tabular}{|c|c|c|c|c|c|c|c|c|c|c|c|c|}
\hline \multirow{3}{*}{$\begin{array}{l}\text { Diagnostic } \\
\text { Year }\end{array}$} & \multicolumn{6}{|c|}{ Including KS and lymphomas } & \multicolumn{6}{|c|}{ Excluding KS and lymphomas } \\
\hline & \multicolumn{3}{|c|}{ Males } & \multicolumn{3}{|c|}{ Females } & \multicolumn{3}{|c|}{ Males } & \multicolumn{3}{|c|}{ Females } \\
\hline & $0-14 y$ & $15-39 y$ & $\geq 40 y$ & $0-14 y$ & $15-39 y$ & $\geq 40 y$ & $0-14 y$ & $15-39 y$ & $\geq 40 y$ & $0-14 y$ & $15-39 y$ & $\geq 40 y$ \\
\hline 1973 & 0.52 & 0.58 & 0.36 & 0.51 & 0.73 & 0.52 & 0.48 & 0.55 & 0.36 & 0.49 & 0.73 & 0.52 \\
\hline 1974 & 0.47 & 0.61 & 0.38 & 0.62 & 0.74 & 0.54 & 0.45 & 0.59 & 0.38 & 0.61 & 0.74 & 0.54 \\
\hline 1975 & 0.52 & 0.64 & 0.39 & 0.61 & 0.74 & 0.55 & 0.51 & 0.61 & 0.39 & 0.61 & 0.73 & 0.55 \\
\hline 1976 & 0.55 & 0.63 & 0.41 & 0.62 & 0.76 & 0.54 & 0.54 & 0.61 & 0.40 & 0.61 & 0.76 & 0.55 \\
\hline 1977 & 0.56 & 0.65 & 0.41 & 0.64 & 0.77 & 0.53 & 0.56 & 0.64 & 0.41 & 0.62 & 0.76 & 0.53 \\
\hline 1978 & 0.61 & 0.64 & 0.41 & 0.65 & 0.77 & 0.54 & 0.62 & 0.63 & 0.41 & 0.65 & 0.76 & 0.54 \\
\hline 1979 & 0.60 & 0.67 & 0.42 & 0.64 & 0.75 & 0.52 & 0.56 & 0.64 & 0.42 & 0.65 & 0.74 & 0.53 \\
\hline 1980 & 0.62 & 0.68 & 0.42 & 0.63 & 0.77 & 0.52 & 0.60 & 0.66 & 0.42 & 0.62 & 0.76 & 0.53 \\
\hline 1981 & 0.61 & 0.69 & 0.43 & 0.69 & 0.76 & 0.53 & 0.60 & 0.68 & 0.43 & 0.68 & 0.76 & 0.53 \\
\hline 1982 & 0.64 & 0.68 & 0.43 & 0.73 & 0.77 & 0.53 & 0.64 & 0.68 & 0.43 & 0.73 & 0.77 & 0.53 \\
\hline 1983 & 0.66 & 0.67 & 0.45 & 0.71 & 0.77 & 0.53 & 0.62 & 0.68 & 0.44 & 0.71 & 0.76 & 0.53 \\
\hline 1984 & 0.66 & 0.61 & 0.44 & 0.66 & 0.77 & 0.54 & 0.63 & 0.65 & 0.44 & 0.66 & 0.77 & 0.55 \\
\hline 1985 & 0.66 & 0.62 & 0.46 & 0.73 & 0.77 & 0.56 & 0.65 & 0.71 & 0.46 & 0.73 & 0.76 & 0.56 \\
\hline 1986 & 0.67 & 0.60 & 0.47 & 0.72 & 0.80 & 0.56 & 0.66 & 0.72 & 0.47 & 0.71 & 0.80 & 0.57 \\
\hline 1987 & 0.70 & 0.58 & 0.50 & 0.71 & 0.80 & 0.57 & 0.69 & 0.72 & 0.50 & 0.71 & 0.79 & 0.57 \\
\hline 1988 & 0.68 & 0.56 & 0.50 & 0.79 & 0.80 & 0.58 & 0.67 & 0.71 & 0.51 & 0.78 & 0.79 & 0.58 \\
\hline 1989 & 0.73 & 0.56 & 0.52 & 0.69 & 0.79 & 0.58 & 0.73 & 0.73 & 0.52 & 0.68 & 0.79 & 0.58 \\
\hline 1990 & 0.78 & 0.57 & 0.55 & 0.69 & 0.79 & 0.59 & 0.76 & 0.73 & 0.55 & 0.68 & 0.79 & 0.59 \\
\hline 1991 & 0.74 & 0.56 & 0.59 & 0.78 & 0.80 & 0.59 & 0.72 & 0.73 & 0.60 & 0.77 & 0.80 & 0.59 \\
\hline 1992 & 0.78 & 0.57 & 0.63 & 0.77 & 0.81 & 0.59 & 0.78 & 0.72 & 0.64 & 0.76 & 0.81 & 0.59 \\
\hline 1993 & 0.73 & 0.59 & 0.61 & 0.80 & 0.82 & 0.59 & 0.72 & 0.75 & 0.62 & 0.79 & 0.82 & 0.59 \\
\hline 1994 & 0.79 & 0.62 & 0.60 & 0.78 & 0.81 & 0.60 & 0.78 & 0.75 & 0.61 & 0.77 & 0.81 & 0.60 \\
\hline 1995 & 0.77 & 0.64 & 0.60 & 0.79 & 0.81 & 0.61 & 0.76 & 0.74 & 0.61 & 0.79 & 0.81 & 0.61 \\
\hline 1996 & 0.80 & 0.71 & 0.62 & 0.81 & 0.83 & 0.61 & 0.79 & 0.77 & 0.62 & 0.80 & 0.83 & 0.61 \\
\hline 1997 & 0.79 & 0.74 & 0.62 & 0.80 & 0.83 & 0.62 & 0.77 & 0.75 & 0.63 & 0.78 & 0.83 & 0.62 \\
\hline 1998 & 0.79 & 0.76 & 0.63 & 0.77 & 0.83 & 0.63 & 0.78 & 0.78 & 0.63 & 0.77 & 0.82 & 0.63 \\
\hline 1999 & 0.77 & 0.76 & 0.65 & 0.82 & 0.85 & 0.63 & 0.75 & 0.77 & 0.65 & 0.82 & 0.84 & 0.63 \\
\hline 2000 & 0.80 & 0.76 & 0.66 & 0.81 & 0.84 & 0.64 & 0.78 & 0.77 & 0.66 & 0.80 & 0.83 & 0.64 \\
\hline 2001 & 0.80 & 0.76 & 0.67 & 0.84 & 0.84 & 0.64 & 0.79 & 0.77 & 0.67 & 0.83 & 0.84 & 0.64 \\
\hline 2002 & 0.80 & 0.79 & 0.67 & 0.83 & 0.85 & 0.65 & 0.79 & 0.79 & 0.67 & 0.83 & 0.85 & 0.65 \\
\hline 2003 & 0.82 & 0.79 & 0.66 & 0.86 & 0.87 & 0.64 & 0.80 & 0.79 & 0.66 & 0.86 & 0.86 & 0.64 \\
\hline 2004 & 0.83 & 0.80 & 0.67 & 0.84 & 0.87 & 0.65 & 0.82 & 0.79 & 0.67 & 0.84 & 0.86 & 0.64 \\
\hline 2005 & 0.82 & 0.82 & 0.67 & 0.82 & 0.86 & 0.66 & 0.81 & 0.82 & 0.67 & 0.82 & 0.85 & 0.65 \\
\hline 2006 & 0.84 & 0.80 & 0.69 & 0.88 & 0.87 & 0.66 & 0.82 & 0.79 & 0.69 & 0.87 & 0.87 & 0.66 \\
\hline 2007 & 0.84 & 0.81 & 0.70 & 0.83 & 0.88 & 0.66 & 0.83 & 0.80 & 0.69 & 0.82 & 0.87 & 0.66 \\
\hline 2008 & 0.84 & 0.82 & 0.69 & 0.83 & 0.89 & 0.67 & 0.84 & 0.82 & 0.69 & 0.81 & 0.88 & 0.66 \\
\hline 2009 & 0.82 & 0.81 & 0.69 & 0.83 & 0.87 & 0.67 & 0.81 & 0.81 & 0.69 & 0.82 & 0.87 & 0.67 \\
\hline Survival ratio 2009 : 1973 & 1.58 & 1.41 & 1.91 & 1.63 & 1.19 & 1.30 & 1.68 & 1.47 & 1.91 & 1.68 & 1.19 & 1.29 \\
\hline
\end{tabular}

between 1983 and 1997 among AYA males due to the onset of the HIV/AIDS epidemic, AYAs of both sexes experienced improved 5-year relative survival throughout 1973-2009. Female AYAs showed consistently higher survival than either male AYAs or females of other age groups (Tables 1-3 and Figures 1-3).

Survival improved from 1973-1977 to 2005-2009 for every age group in both sexes (Table 4 and Figure 4, A, B). Although age-specific 5-year relative survival improved by $21 \%-49 \%$ for male AYAs and 13\%-20\% for female AYAs, their magnitudes of improvement are smaller than those for children $(57 \%-61 \%$ for males and $35 \%-43 \%$ for females) and older adults (50\%-85\% for males and $23 \%-25 \%$ for females) (Table 4). As a result, although AYAs with cancer consistently maintained the highest survival in both time periods, their survival superiority during 1973-1977 diminished substantially by 2005-2009, as survival improvement in both children and older adults outpaced that of AYAs (Figure 4, A, B).

\section{Discussion}

We evaluated 5-year relative survival by sex and age over time among patients diagnosed with invasive cancer during 19732009 from the SEER 9 registries, a population-based source representing the experience throughout the United States. These data covered the time periods before, during, and following the HIV/AIDS epidemic, allowing us to observe the impact of HIV/ AIDS on cancer survival through inclusion and exclusion of KS and lymphomas, two major HIV/AIDS-associated malignancies. We found superior cancer survival among AYAs of both sexes throughout the 1970s as compared to younger and older patients, which was also noted by an earlier report (7). However, such survival advantage disappeared in AYA males during the 1980s-1990s, a time associated with the burgeoning HIV/AIDS epidemic in the United States. By excluding KS and lymphomas from the sex-age-specific analysis, we were able to show that this observed decline in cancer survival among AYAs was (1) 

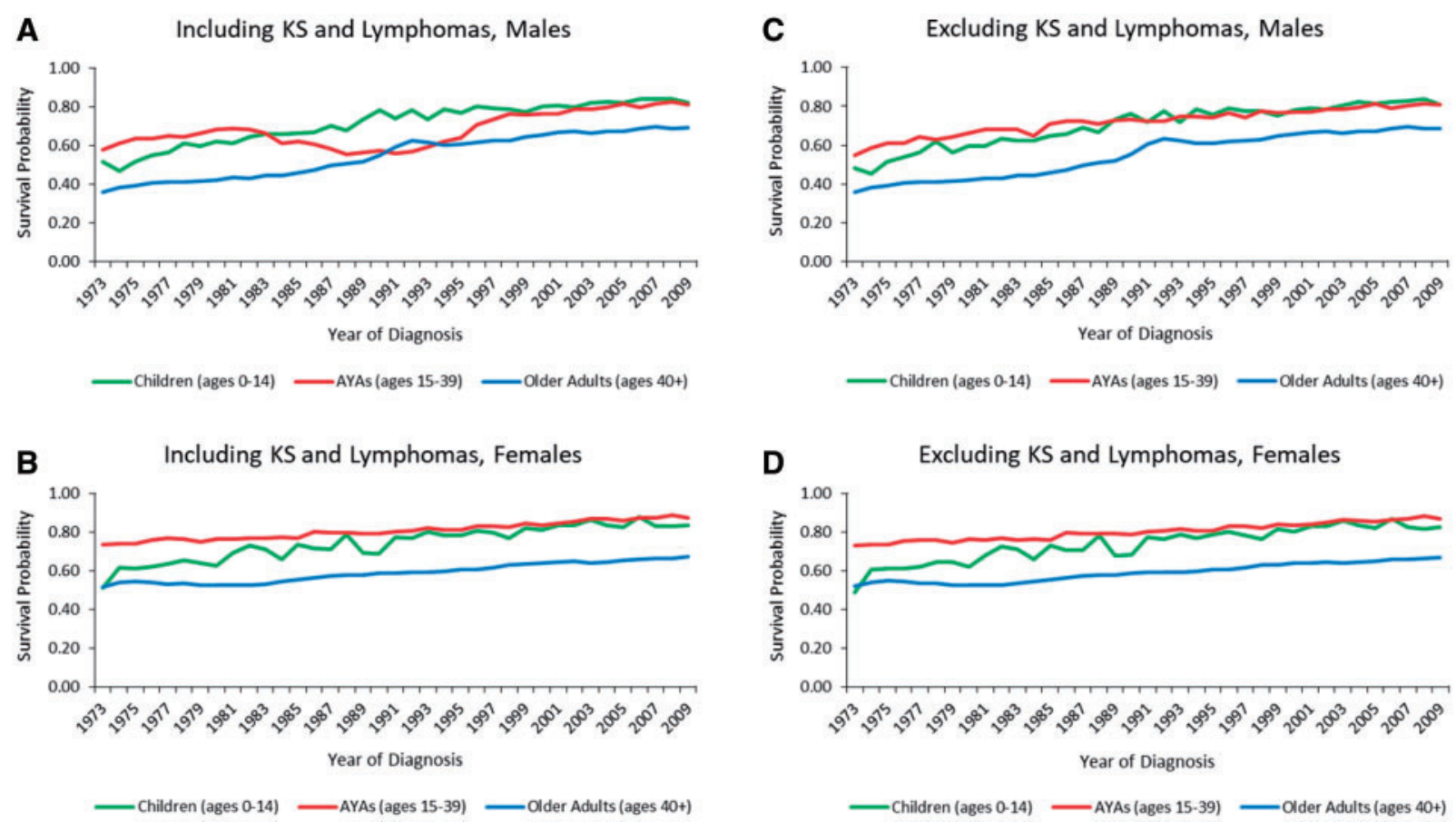

Figure 3. Time trends of 5-year relative survival by age group and sex. Data were from the SEER 9 registries, 1973-2009. Analyses for probability of survival from all invasive cancers including Kaposi sarcoma (KS) and lymphomas are shown for (A) males and (B) females. Analyses for probability of survival from all invasive cancers excluding KS and lymphomas are shown for (C) males and (D) females. AYA = adolescents and young adults.

Table 4. Five-year relative survival by sex, age group, and time period, all invasive cancers, SEER 9 registries*

\begin{tabular}{|c|c|c|c|c|c|c|c|}
\hline \multirow{3}{*}{$\begin{array}{l}\text { Population } \\
\text { groups }\end{array}$} & \multirow[b]{3}{*}{ Age groups, y } & \multicolumn{4}{|c|}{ 5-Year relative survival } & \multirow{2}{*}{\multicolumn{2}{|c|}{$\begin{array}{c}\text { Survival ratio } \\
2005-2009: 1973-1977\end{array}$}} \\
\hline & & \multicolumn{2}{|c|}{ 1973-1977 } & \multicolumn{2}{|c|}{ 2005-2009 } & & \\
\hline & & Males & Females & Males & Females & Males & Females \\
\hline \multirow[t]{3}{*}{ Children } & $0-4$ & 0.52 & 0.59 & 0.83 & 0.84 & 1.61 & 1.43 \\
\hline & $5-9$ & 0.53 & 0.59 & 0.83 & 0.80 & 1.58 & 1.35 \\
\hline & $10-14$ & 0.52 & 0.63 & 0.82 & 0.87 & 1.57 & 1.38 \\
\hline \multirow[t]{5}{*}{ AYAs } & 15-19 & 0.56 & 0.74 & 0.83 & 0.88 & 1.49 & 1.18 \\
\hline & $20-24$ & 0.64 & 0.78 & 0.83 & 0.89 & 1.30 & 1.14 \\
\hline & $25-29$ & 0.69 & 0.79 & 0.83 & 0.89 & 1.21 & 1.13 \\
\hline & $30-34$ & 0.65 & 0.75 & 0.82 & 0.88 & 1.26 & 1.17 \\
\hline & $35-39$ & 0.57 & 0.72 & 0.78 & 0.86 & 1.38 & 1.20 \\
\hline \multirow{5}{*}{$\begin{array}{l}\text { Older } \\
\text { adults }\end{array}$} & $40-44$ & 0.50 & 0.68 & 0.75 & 0.85 & 1.50 & 1.24 \\
\hline & $45-49$ & 0.42 & 0.66 & 0.71 & 0.82 & 1.68 & 1.24 \\
\hline & $50-54$ & 0.40 & 0.63 & 0.70 & 0.78 & 1.78 & 1.24 \\
\hline & $55-59$ & 0.39 & 0.60 & 0.72 & 0.74 & 1.85 & 1.25 \\
\hline & $\geq 60$ & 0.39 & 0.48 & 0.67 & 0.59 & 1.75 & 1.23 \\
\hline
\end{tabular}

${ }^{*} \mathrm{AYA}=$ adolescents and young adults.

negated; (2) principally limited to AYA males who were disproportionately burdened by HIV/AIDS in the United States $(14,15)$; and (3) transient, with resolution by the early 2000s after cART entered wide clinical use, providing highly effective treatment for HIV/AIDS $(14,15)$.

Our analysis demonstrated that cancer survival has been rising in all age groups for both males and females including AYAs. In fact, AYAs consistently exhibited better survival than other age groups when HIV/AIDS-related cancers were excluded and after HIV/AIDS was effectively controlled. This finding concurs with a recent report that concluded AYAs have generally good outcomes after cancer diagnosis, noting their better overall health, greater ability to recover from cancer treatment, and lower mortality from other causes compared to older adults (16). Across age groups, females consistently displayed better survival than males, which was important information that was previously masked when the analysis was not sex specific (5).

Our analysis does indicate that the magnitude of survival improvement was smaller in AYAs than in either children or older adults and smaller in females than in males. This is explained by a combination of higher baseline survival of both AYAs and females, respectively, and more substantial increases in survival in both children and older adults (illustrated in Figure 4). Special emphasis on childhood cancer since the 1950 s 

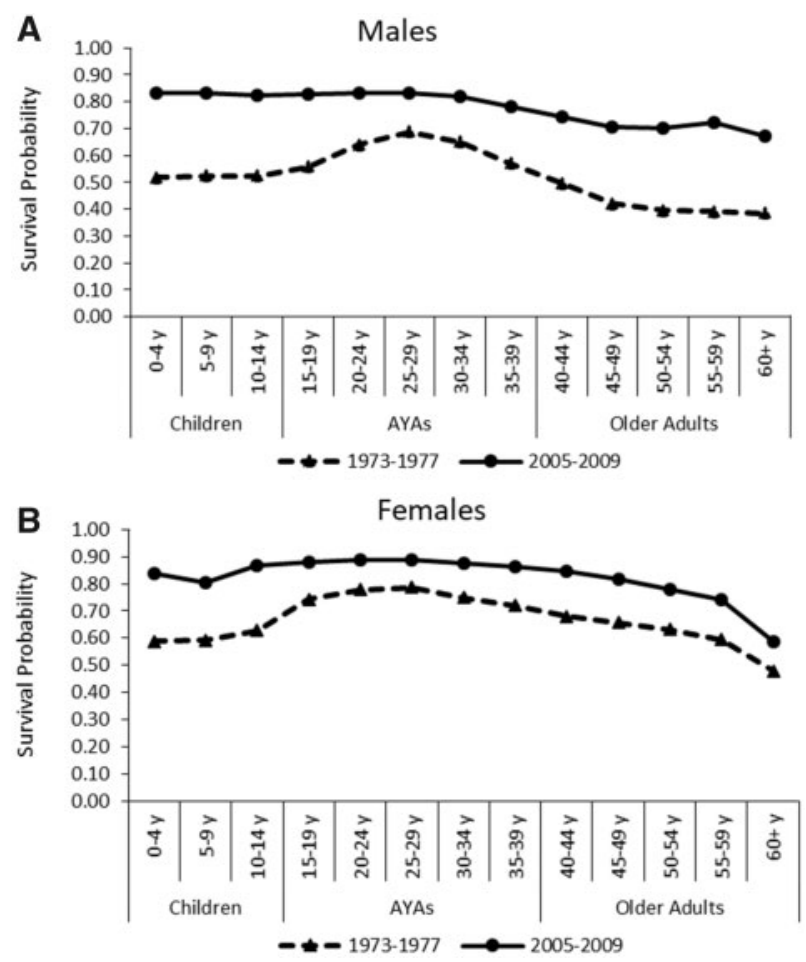

Figure 4. Comparison of 5-year relative survival for all invasive cancers by sex and age group, 1973-1977 vs 2005-2009. Data from the SEER 9 registries, 19732009, were analyzed. Age-specific 5-year relative survival during 1973-1977 and 2005-2009 are plotted separately for (A) males and (B) females. AYA = adolescents and young adults.

and passage of the National Cancer Act of 1971 have been credited for the dramatic improvement in cancer survival in children and older adults, respectively $(3,5,7)$. Hence, in analyzing comparative survival trends for AYAs, it is important to be cognizant that less relative improvement does not necessarily correspond to poorer absolute survival.

These findings support our hypothesis and provide both an explanation for the historical observation of inferior survival improvement for AYAs and evidence that AYA cancer survival is, in fact, largely superior to other age groups and steadily improving. In addition, our findings reveal at least two more insights. First, reporting survival improvement alone, whether it is measured by absolute or relative differences in survival probabilities between two time periods or by annual percent change in survival, provides only partial information for evaluating progress. Actual survival probabilities by year or time period supplement measures of survival improvement to form a more complete assessment. Second, cancer risk and survival differ not only by age, but also by sex, particularly among AYAs. Therefore, informative assessment of survival should be sex specific as well. These two insights help ensure the most complete, accurate, and informative cancer survival data are available to aid the development of research and treatment priorities, and to direct resources most appropriately.

In reevaluating the previously reported poorer survival improvement in AYA cancer patients in comparison with other age groups considering the impact of HIV/AIDS, we followed a similar analytical scope as used in the previous report, although we added the age-specific analysis. This approach brought about two limitations of our study: We only examined survival at 5 years after diagnosis, and for all cancers combined. Challenges associated with long-term survival after 5 years are of particular importance for AYAs as they move through the different stages of life. The aggregate survival of all cancers combined does not necessarily represent or reveal survival of specific cancer types. Future efforts focusing on the long-term survival experience by cancer type will likely produce valuable new information.

Our analysis conveys a hopeful message that overall cancer survival among AYAs continues to exceed that of other age groups. In this sense, the future for AYA oncology looks bright. However, this does not negate other serious challenges facing this population, including differences in cancer and host biology, impaired access to appropriate cancer treatment, low participation in cancer clinical trials, increased susceptibility to acute and long-term treatment-related toxicity, and the need for intensive psychosocial support and long-term survivorship care. Additional AYA-focused cancer-specific research is needed in these areas to gain a better understanding of factors influencing cancer-specific outcomes and long-term survival.

\section{Funding}

The collection of cancer incidence data used in this study was supported by the California Department of Public Health pursuant to California Health and Safety Code Section 103885; Centers for Disease Control and Prevention's (CDC) National Program of Cancer Registries, under cooperative agreement 5NU58DP003862-04/DP003862; the National Cancer Institute's Surveillance, Epidemiology and End Results Program under contract HHSN261201000140C awarded to the Cancer Prevention Institute of California, contract HHSN261201000035C awarded to the University of Southern California, and contract HHSN261201000034C awarded to the Public Health Institute. The work by Dr Moke on this project was supported by training grants from the National Cancer Institute of the National Institutes of Health under contract T32CA09659 awarded to Children's Hospital Los Angeles. The work by Dr Deapen and Dr Freyer was also in part supported by award number P30CA014089 from the National Cancer Institute.

\section{Notes}

Affiliations of authors: Los Angeles Cancer Surveillance Program, Department of Preventive Medicine, Keck School of Medicine, University of Southern California, Los Angeles, CA (LL, KYT, AH, ASH, JZ, MC, DD); Children's Center for Cancer and Blood Diseases, Children's Hospital Los Angeles, Los Angeles, CA (DJM, DRF); USC Norris Comprehensive Cancer Center, University of Southern California, Los Angeles, CA (AH, DRF, MC, DD); University of Colorado Comprehensive Cancer Center, University of Colorado, Denver, CO (MC).

The funders had no role in the design of the study; the collection, analysis, and interpretation of the data; the writing of the manuscript; and the decision to submit the manuscript for publication.

The authors have no conflicts of interest to disclose.

The ideas and opinions expressed herein are those of the authors and do not necessarily represent the official views of the State of California, Department of Public Health, the Centers for Disease Control and Prevention, the National Cancer Institute, the National Institutes of Health, or their contractors and subcontractors. This work would not be possible without 
the hard work and dedication of cancer registrars and staff across all SEER registries.

\section{References}

1. Siegel RL, Miller KD, Jemal A. Cancer statistics, 2018. CA Cancer J Clin. 2018; 68(1):7-30.

2. National Cancer Institute. Adolescents and Young Adults With Cancer. https:// www.cancer.gov/types/aya. Accessed April 12, 2018.

3. Bleyer A, Barr R, Hayes-Lattin B, Thomas D, Ellis C, Anderson B; Biology and Clinical Trials Subgroups of the US National Cancer Institute Progress Review Group in Adolescent and Young Adult Oncology. The distinctive biology of cancer in adolescents and young adults. Nat Rev Cancer. 2008;8(4):288-298.

4. Bleyer A, Budd T, Montello M. Adolescents and young adults with cancer. Cancer. 2006;107(7 suppl):1645-1655.

5. Adolescent and Young Adult Oncology Progress Review Group. Closing the Gap: Research and Care Imperatives for Adolescents and Young Adults With Cancer. U.S. Department of Health and Human Services, National Institutes of Health National Cancer Institute, LIVESTRONG Young Adult Alliance; 2006. NIH Pub. No. 06-6067. https://www.cancer.gov/types/aya/research/ayao-august-2006. pdf. Accessed April 12, 2018.

6. Bleyer A. Latest estimates of survival rates of the 24 most common cancers in adolescent and young adult Americans. J Adolesc Young Adult Oncol. 2011;1(1):37-42.

7. Keegan TH, Ries LA, Barr RD, et al; National Cancer Institute Next Steps for Adolescent and Young Adult Oncology Epidemiology Working Group. Comparison of cancer survival trends in the United States of adolescents and young adults with those in children and older adults. Cancer. 2016;122(7): 1009-1016.
8. Detels R, Tarwater P, Phair JP, Margolick J, Riddler SA, Muñoz A. Effectiveness of potent antiretroviral therapies on the incidence of opportunistic infections before and after AIDS diagnosis. AIDS. 2001;15(3):347-355.

9. Palella FJ, Delaney KM, Moorman AC, et al. Declining morbidity and mortality among patients with advanced human immunodeficiency virus infection. $\mathrm{N}$ Engl J Med. 1998;338(13):853-860.

10. Surveillance, Epidemiology, and End Results (SEER) Program (www.seer.cancer.gov) SEER*Stat Database: Incidence-SEER 9 Regs Research Data, Nov 2016 Sub (1973-2014) < Katrina/Rita Population Adjustment>-Linked To County Attributes - Total U.S., 1969-2015 Counties, National Cancer Institute, DCCPS, Surveillance Research Program, released April 2017, based on the November 2016 submission.

11. Calza L, Manfredi R, Colangeli V, Dentale N, Chiodo F. Hodgkin's disease in the setting of human immunodeficiency virus infection. Scand J Infect Dis. 2003;35(2):136-141.

12. Chiu BC, Weisenburger DD. An update of the epidemiology of non-Hodgkin's lymphoma. Clin Lymphoma. 2003;4(3):161-168.

13. SEER Program. Relative Survival, SEER*Stat Web Help. https://seer.cancer.gov/ seerstat/WebHelp/Relative_Survival.htm. Accessed March 19, 2018.

14. Jones J, Salazar L. A historical overview of the epidemiology of HIV/AIDS in the United States. In: Wright ER, Carnes N, eds, Understanding the HIV/ AIDS Epidemic in the United States, the Role of Syndemics in the Production of Health Disparities. Switzerland: Springer International Publishing; 2016: $19-41$.

15. Centers for Disease Control and Prevention. HIV Among Men in the United States. U.S. Department of Health \& Human Services. https://www.cdc.gov/ hiv/group/gender/men/index.html. Accessed March 19, 2018.

16. Lewis DR, Seibel NL, Smith AW, Stedman MR. Adolescent and young adult cancer survival. J Natl Cancer Inst Monogr. 2014;2014(49):228-235. 